\title{
Associations between use of self-regulatory strategies and daily eating patterns: An experience sampling study in college-aged women
}

\author{
Richard B. Lopez ${ }^{1}$ [ $\cdot$ Danielle Cosme $^{2} \cdot$ Kaitlyn M. Werner ${ }^{3} \cdot$ Blair Saunders $^{4} \cdot$ Wilhelm Hofmann $^{5}$
}

Accepted: 9 July 2021 / Published online: 15 July 2021

(c) The Author(s) 2021

\begin{abstract}
Previous theorizing suggests there are multiple means by which people regulate their emotions and impulses, but that these strategies vary in the degree to which they support goal attainment. Some have proposed that proactive strategies (e.g. situation selection, distraction) may be particularly effective, while interventive strategies (e.g. suppression) are less effective. Despite these diverging predictions, researchers have yet to examine spontaneous use of these strategies and their respective and combined efficacy when applied to momentary food desires experienced in daily life. In the present study, we assessed eating patterns for one week via ecological momentary assessment in college-aged women $(\mathrm{N}=106)$. Results from preregistered analyses indicated that using a variety of strategies, including preventative strategies such as situation selection and distraction, was associated with greater self-control success, as indexed by weaker desires, higher resistance, lower likelihood of enacting desires, and less food consumed. A similar pattern was observed when participants implemented additional strategies during desire episodes, which they were more likely to do when their desires conflicted with other self-regulatory goals. All associations were observed while controlling for momentary hunger levels, dieting status, age, and body mass index. These findings are consistent with a growing body of work assessing people's spontaneous use of emotion regulation strategies in everyday contexts, suggesting potential meta-motivational tendencies marked by flexible and adaptive use of self-regulatory strategies.
\end{abstract}

Keywords Self-regulation $\cdot$ Emotion regulation $\cdot$ Self-control $\cdot$ Strategies $\cdot$ Eating behavior $\cdot$ EMA

The human capacity for goal pursuit and self-regulation, especially when foregoing short term pleasures in the service of long term, not-yet-attained goals, is one of our species' most impressive-yet sometimes fickle—abilities. Successful goal pursuit has positive downstream consequences for an individual's self-confidence, health, and well-being, but perhaps self-regulation of daily eating behavior has some

Richard B. Lopez

rlopez@bard.edu

1 Department of Psychology, Bard College, Annandale-on-Hudson, NY 12504, USA

2 Annenberg School for Communication, University of Pennsylvania, Philadelphia, PA, USA

3 Department of Psychology, University of Toronto, Toronto, Canada

4 School of Social Sciences, University of Dundee, Dundee, Scotland, UK

5 Department of Psychology, Ruhr University Bochum, Bochum, Germany of the most obvious and direct impacts on health. Indeed, the global obesity epidemic shows no signs of slowing (Friedrich, 2017), and unregulated patterns of unhealthy eating pose additional health risks, such as higher likelihood of developing diabetes (Patti \& Kahn, 2004) and cancer (O'Leary et al., 2018). Thus, it is important to understand the mechanisms underlying daily eating behavior, with a special focus on regulatory strategies that are associated with healthier eating patterns, which not only provide nutritional benefits, but can also reduce the risk of obesity and other health conditions.

Although the effectiveness of different regulatory strategies has been of interest to psychologists for decades (see Duckworth et al., 2018 for a review; also; Gollwitzer, 1999; Rodriguez et al., 1989), research on self-control has largely focused on particular regulatory strategies and their efficacy, in isolation. Initially, studies were mainly concerned with identifying the limits of a putative, domain-general capacity to exert control in the first place, as posited by the ego depletion model of self-control (Baumeister et al., 1998). 
This model has since been critiqued in light of large-scale replication studies that have, at best, provided weak evidence for ego depletion effects (Dang et al., 2020; Friese et al., 2018; Hagger et al., 2016). More recently, instead of conceptualizing self-regulation as arising exclusively from exertion of limited willpower, researchers have begun to identify and examine relevant contributing factors, including situation-level and person-level factors, that may be more (or less) conducive to successful goal pursuit (Fujita, 2011; Gillebaart \& de Ridder, 2015; Hennecke \& Bürgler, 2020; Hofmann \& Kotabe, 2012).

According to the process model of self-control, when a person encounters a temptation that is at odds with their goals (e.g. eating a brownie sundae while dieting), they are more likely to successfully stick to their goal by using selfregulatory strategies earlier in the process of anticipating or responding to the temptation than those that occur later, after the temptation has intensified (Duckworth et al., 2016). For decades, classic tests of self-control, such as the marshmallow test, have revealed that individuals use a range of strategies to overcome immediate temptations, such as engaging in non-consummatory thoughts about the food (e.g. imagining a marshmallow as a fluffy cloud); thinking of distracting positive thoughts; or moving the temptation out of sight (see Metcalfe \& Mischel, 1999 for a review).

The process model of self-control integrates these findings, and further predicts that earlier strategies such as modifying a situation that presents someone with an impulse-eliciting temptation (e.g. by moving or removing the tempting food item) are theorized to be most effective (Duckworth et al., 2018). In contrast, strategies that are applied later in the impulse generation process, such as suppression, are hypothesized to be less effective. The rationale is that it may be easier to apply a more proactive strategy that minimizes the likelihood of ever experiencing a strong temptation in the first place (e.g. avoiding the kitchen where there are freshbaked cookies), versus applying a reactive strategy once an impulse and its behavioral ramifications are completely experienced (e.g. suppressing your desire for the cookies; for a similar argument, see Hofmann \& Kotabe, 2012). This supposition is supported by a recent experience sampling study (Williamson \& Wilkowski, 2020), which found that proactive strategies were more reliably associated with goal progress than more reactive strategies (e.g. inhibition or suppression).

While most research stemming from the process model and strategy use more generally has focused on comparing the effectiveness of individual strategies, it is likely that people use more than one strategy within the same regulation episode. Indeed, recent theorizing suggests that the use of multiple strategies-a form of polyregulation - may be particularly adaptive and facilitate goal pursuit (Ford et al., 2019; Werner \& Ford, 2021). For example, a person can hide the cookies that their spouse baked (situation modification) while at the same time thinking about how much of a calorie bomb it would be to eat the cookies (cognitive change).

Building on the process model of self-control (Duckworth et al., 2016) and the concept of polyregulation, the overall aim of the present study was to examine spontaneous (i.e. uninstructed) use of multiple regulatory strategies and the respective and combined efficacy of these strategies when applied to food desires people experience in daily life. Thus, we sought to address several questions.

Hypothesis 1: Strategy type According to the process model, how do specific strategies that occur earlier versus later in the impulse-generation cycle relate to (un)successful regulation of momentary food desires - as measured by desire strength, resistance, desire enactment, and/or amount of food consumed? We first hypothesize that participants will generally experience greater self-control success when applying relatively more proactive strategies (e.g. situational selection, mindfulness, and distraction), relative to more reactive strategies, such as thought suppression.

Hypothesis 2: Polyregulation With respect to polyregulation, the present study asks: is the use of multiple desire regulation strategies per episode associated with greater success, as has been shown in the affective domain (Blanke et al., 2020; Grommisch et al., 2019; Southward \& Cheavens, 2020)? We hypothesize that greater polyregulation (i.e. the number of strategies used) will be positively associated with self-control success, as operationalized by any of the following: weaker desires, higher resistance, less frequent enactment of reported desires, and/or less food consumed when desires are enacted.

Hypothesis 3: Goal pursuit Given the variability in motivational factors during people's real world goal pursuit (Milyavskaya \& Werner, 2018), especially the effects of goal conflict on self-regulatory processes (Hofmann et al., 2012), we were interested in relations between goal-related processes and the selection and/or efficacy of strategy use. Goal conflict is a critical and necessary precursor to self-control because it signals the need to regulate, which could prompt use of multiple regulatory strategies, whereas an absence of conflict indicates that the food desire does not rise to the level of a temptation that compromises one's goal pursuit, so there is no need to regulate (and therefore no need to implement regulatory strategies; see Hofmann \& Kotabe, 2012 and Werner \& Ford, 2021 for further discussion on this topic). Thus, we hypothesize that for participants whose food desires conflict with other goals they are pursuing (e.g. dieting), there will be higher resistance to desires and employment of additional self-regulatory strategies, which will in turn be associated with less frequent enactment of desires. 
To test the aforementioned hypotheses, we conducted pre-registered and exploratory secondary analyses on a sample of college-aged women $(n=106)$, whose eating patterns were assessed for one week via ecological momentary assessment (EMA). For each EMA signal, participants reported whether or not they currently or recently experienced a desire for food. If so, they reported the strength of the desire, whether the desire conflicted with other goals they had, how much they resisted, whether or not they enacted the desire, and how much of the desired food they consumed. For each desire episode, we also asked participants to report whether they applied any self-regulatory strategies, which mapped relatively well onto different stages of the impulse-generation process, as per the process model (see Method below for additional details about the EMA procedure). Self-regulatory success was defined as moments when participants reported weaker desires, greater resistance to those desires, lower likelihood of desire enactment, and lower consumption of desired foods. We expect the following will be associated with success: (1) Greater use of proactive/earlier strategies (vs. reactive/later strategies); (2) Use of additional strategies during a desire episode; and (3) Goal-related processes experienced in the moment, specifically goal conflict, via direct associations with outcome measures and indirect associations via polyregulation (i.e. use of multiple/additional strategies).

\section{Statement of transparency}

We conducted secondary analyses on data that were collected as part of two separate projects $(n=31, n=75)$ that tested independent hypotheses pertaining to brain-behavior relationships in the eating domain that have been previously reported (Cosme \& Lopez, 2020; Lopez et al., , 2014, 2017). Since we are using secondary data, the current study design was not preregistered; however, we preregistered the analytical plan for this particular paper prior to conducting any analyses (https://osf.io/pfwra). Deviations from the preregistered analysis plan include: (1) conducting exploratory analyses to test Hypothesis 1 using contrast coding to explicitly compare earlier versus later strategies, and situational/externally oriented versus other strategies; and (2) operationalizing polyregulation using a count variable rather than an ordinal variable to test Hypothesis 2. Further details regarding the rationale for these deviations are reported in footnotes. All data and R code used for the present analyses are posted on the Open Science Framework (project link: https://osf.io/mtpje/).

\section{Method}

\section{Participants and procedure}

All participants were recruited from the Dartmouth community and were eligible to participate if they: (1) were at least 18 years of age; and (2) reported no history of neurological problems or psychiatric disorders. There were 106 women $\left(\mathrm{M}_{\text {age }}=19.9 ; \mathrm{SD}_{\text {age }}=1.9 ;\right.$ range $\left.=18-28\right)$ who met these eligibility criteria and whose data were used for all subsequent analyses. Of these participants, $59.4 \%$ identified as being White, $17.8 \%$ identified as being Asian, Asian American, or Pacific Islander, 8.9\% identified as being American Indian or Alaskan Native, 7.9\% identified as being Black or African American, and 5.9\% identified as being Biracial or Multi-racial. All participants gave informed consent according to guidelines set by Dartmouth's Committee for the Protection of Human Subjects. Upon completion of the study, participants were debriefed on the study's overall aim to better understand self-regulatory behaviors in the eating domain.

First, participants came into the lab to complete personality questionnaires and provide height and weight measurements (to estimate body mass index, see below). At this time, they also underwent an fMRI scanning session in which they completed a food cue reactivity task (as part of a larger set of studies, as mentioned above; neuroimaging data are reported elsewhere). Next, they enrolled in a week-long sampling of their daily eating behaviors, which were captured via an EMA protocol consisting of seven surveys administered at random intervals across a 14-h window each day (see Hofmann et al., 2012; Lopez et al., 2014 for complete details of this protocol). The EMA surveys included assessment of self-regulatory strategy use, which served as key predictors in our pre-registered analyses, as well as different facets of eating behaviors that served as criterion measures of interest (see below for all measures described in greater detail).

\section{Assessment of daily eating behaviors via EMA}

For each signal during the EMA, participants first responded to the question, "Do you currently have a desire for food, or have you had a recent desire for food within the last $20 \mathrm{~min}$ ?" If they reported having a current or recent desire, additional questions appeared that assessed desire strength ("How strong is/was your desire (at its peak) to eat the food?"; reported on a scale of $0-6$, where $0=$ no desire at all and $6=$ irresistible), and level of resistance ("How much did you try to resist this desire?"; reported on a scale of 0-6, where $0=$ not at all and $6=$ very much). 
If participants reported exerting at least a little resistance ( $\geq 1$ on the $0-6$ scale), they were asked, "How did you try to resist?" and could indicate any of the following, as many that applied: suppression ("by suppressing thoughts about the food"); distraction ("by distracting myself with something else"); mindful self-monitoring ("by monitoring closely what I'm doing”); goal recollection ("by reminding myself of other goals I have"); situation modification ( "by removing the food from my immediate environment"); situation selection ("by leaving the situation"); acceptance ("by simply accepting my desire for the food"); interpersonal accountability ("by promising someone I won't touch the food"); and an open-ended response option: "Other (please specify)." Participants were also asked whether they enacted the desire ("Did you already consume some of the food you desire(d)?") and, if they did enact the desire, how much they consumed ("How much did you eat?"; reported on a scale of 0-6, where $0=$ nothing at all and $6=$ way more than a regular portion/I'm stuffed).

In addition to the above measures, we also measured aspects of participants' goal pursuit, including level of conflict with other goals they may have had ("How much does eating this food conflict with other important goal(s) you may have?"; reported on a scale of $0-6$, where $0=$ not at all and $6=$ very much), and goal importance ("How important, taken together, are these goals to you?"; reported on a scale of $0-6$, where $0=$ not at all and $6=$ very much).

\section{Covariates}

\section{Level 1 (observation-level) covariates}

In addition to the measures above, participants' momentary hunger levels were assessed for every EMA signal ("How hungry are you at the moment?", reported on a scale of 0-6, where $0=$ not at all and $6=$ very hungry).

\section{Level 2 (person-level) covariates}

To assess individual differences in participants' dieting tendencies, we included sum scores from the 10-item Revised Restraint Scale, which captures two important aspects of dieting behavior, namely: concern for dieting and weight fluctuations (Heatherton et al., 1988; Herman \& Polivy, 1980). We also assessed participants' body mass index (kg/ $\mathrm{m} 2$ ) and body fat percentage via the Tanita Total Body Composition Analyzer (model TBF-300A; Tanita Corporation, Arlington Heights, IL, USA). This is an acceptable method to estimate body composition and is comparable to other techniques that use bioelectrical impedance to estimate various metrics of body composition, including total body water and body fat percentage (Jebb et al., 2000).

\section{Power and effect size considerations}

Although there were no formal a priori power calculations conducted for the present analyses, we followed recent recommendations outlined by the SPSP Power Analysis Working Group (Giner-Sorolla et al., 2019) by conducting a sensitivity analysis, which estimates the minimum population effect size detectable at (or above) a desired level of statistical power, given the sample size. Because most of our hypotheses were about direct effects at the observation level (level 1), we consulted results from Monte Carlo simulations conducted by Arend and Schäfer (2019) to determine minimum detectable effect sizes given the number of observations at level $1\left(\mathrm{M}_{\mathrm{obs}}=34.9 ; \mathrm{SD}_{\mathrm{obs}}=9.18\right.$; range $\left.=8-48\right)$ and level $2(\mathrm{~N}=106)$ (Arend \& Schäfer, 2019). With $\geq 80 \%$ power and across a range of small to large intraclass correlations, the present sample allows for detection of standardized effect sizes $(\gamma)$ as small as 0.09 (see Table 5 in Arend \& Schäfer, 2019).

\section{Statistical analyses and model specifications}

First, we computed counts and proportions corresponding to the number of strategies used to resist experienced desires (for each desire episode, as well as the average number of strategies used across all reported desire episodes for each participant).

Next, we fit several multilevel models to predict participants' momentary experiences of food desire, resistance, whether or not they enacted a reported desire, and the quantities of food they reported consuming. Following our preregistration, we coded type of strategy according to earlier versus later stages and processes proposed by the process model of self-control (Duckworth et al., 2016), specifically: (1) leaving the situation, removing the food from my immediate environment, and promising someone I won't touch the food were categorized as situational (externally oriented) strategies; (2) distraction served as its own category, as it was the only strategy here that involved attentional deployment; (3) monitoring closely what I'm doing, reminding myself of other goals I have, and accepting my craving were considered cognitive strategies; and (4) suppression served as its own category, as it was the only strategy involving response-modulation. These strategy groupings are consistent with previous theorizing and other experience sampling studies that have taken similar approaches to conceptualizing regulatory strategies along a dimension ranging from earlier, more preventative strategies to later, more interventive ones (e.g. Hennecke et al., 2019; Hofmann \& Kotabe, 2012).

Hypothesis 1: Strategy type First, to be consistent with what we indicated in our preregistration and to examine each strategy's individual efficacy, we ran a set of models in which 
we included separate dummy-coded dichotomous predictors indicating which type of the four abovementioned regulatory strategies, if any, participants used during each desire episode. Importantly, in these two sets of models (i.e. with strategy type as an ordinal variable and multiple strategies as separate predictors) we also incorporated use of other/ idiosyncratic strategies, which participants could report with the free-response option. Some of these other strategies included swapping an unhealthy for a healthy option (e.g. "By eating fruit instead"), drinking water, or being unable to enact to the desire for various reasons (e.g. "Running to a meeting" or "Being in a car so unable to get it"). These strategies occurred infrequently compared to the others (i.e. $4.2 \%$ of observations versus $26.1 \%$ for distraction and $30.1 \%$ for cognitive strategies) and although we had no a priori hypotheses about idiosyncratic strategies, we thought it would be important to include an additional dummy-coded variable to control for idiosyncratic strategy use.

Next, to explicitly test predictions from the process model, strategy type was represented by an ordinal variable indicating the earliest regulatory strategy (as per the process model) participants reported using during each desire episode (if any), with the variable coded as follows: 1 represented externally oriented strategies, 2 represented distraction, 3 represented cognitive strategies, and 4 represented suppression. This allowed us to examine relative efficacy of these strategies and specify contrasts to directly test our first hypothesis, that earlier, more proactive strategies would be more effective than later, more reactive strategies. ${ }^{1}$ Accordingly, we used a polynomial contrast for the ordinal strategy variable, which could reveal potential linear effects, as well as a custom contrast comparing externally-oriented strategies to all others, given such strategies have been relatively understudied and have been hypothesized to hold particular promise to promote sustainable self-regulatory patterns (Duckworth et al., 2016).

Hypothesis 2: Polyregulation To test whether using multiple strategies is positively correlated with greater self-regulatory

\footnotetext{
1 Although this hypothesis and its rationale was preregistered, specifying an ordinal variable was not explicitly mentioned in our pre-registration. In the spirit of transparency, and as we did with operationalizations of number of strategies (see footnote 2, below), we report two approaches here (i.e., strategy use as an ordinal variable, and strategies as multiple, dummy-coded variables), which taken together are a more comprehensive test of our preregistered hypothesis. Indeed, the results generally converge to guide inferences about particular strategies (e.g., when pitted against other strategies, situational/externally-oriented strategies are more effective to reduce amount eaten, which is recapitulated in the companion model with dummy-coded variables that show the proactive strategies variable having among the lowest regression coefficients compared to other strategies)
}

success, we fit a companion set of models in which we treated number of strategies as a count variable ${ }^{2}$ and fit the models with the same four outcomes measures.

Hypothesis 3: Goal pursuit To determine whether momentary variation in goal-related processes (i.e. goal conflict and importance) is associated with self-regulatory success, via use of multiple strategies, we fit additional multilevel mediation models with goal conflict and goal importance as predictors, number of strategies entered as the mediator, and enactment of desires and amount eaten as outcome measures.

For all statistical models, we included additional variables to control for their potential influence on the criterion (outcome) measures, as per our preregistration. Specifically, person-level (Level 2) covariates included a dichotomous variable indicating chronic dieting status, as well as age and body mass index (BMI). We also included momentary experience of hunger at the observation-level (Level 1). Dietary restraint is an individual difference measure that commonly impacts self-regulatory processes in lab contexts and daily life (e.g. Lopez et al., 2016; Wagner et al., 2013). We used the Revised Restraint Scale, which can determine dieting status (Heatherton et al., 1988, 1991; Herman \& Polivy, 1980), with participants required to report a composite score of 16 or greater (across both "Concern for Dieting" and "Weight Fluctuation" subscales) to be classified as a chronic dieter. Next, we included age to account for individual differences in development and maturation of executive functions important for self-regulation, including inhibitory control (Hofmann, 2017). BMI was included to account for biological factors that may affect and reflect eating behavior tendencies. Lastly, momentary hunger levels were included at Level 1 to control for any consummatory behavior arising from needs for homeostatic eating.

All continuous Level 1 predictors were person-mean centered and all Level 2 predictors were grand-mean centered. Unless otherwise specified, all multilevel models were fit with random intercepts. All data analysis and modeling were conducted in $\mathrm{R}$, with multilevel models specified and run via the lme4 package, version 1.1-23 (Bates et al., 2014). Bayesian multilevel mediation models were fit using the brm()

\footnotetext{
${ }^{2}$ Originally, we operationalized this variable as an ordinal variable with three levels indicating no strategies, a single strategy, or multiple strategies applied during each desire episode. In a deviation from the preregistration, we realized that a count variable would serve as a better translation of our hypothesis and lead to more interpretable results, so that is what is reported in the main text. For the sake of thoroughness and transparency, we have included results using the ordinal variable in the supplement. The results are very similar and inferences about the efficacy of number of strategies do not appreciably change whether the variable is a count or ordinal variable.
} 
function from the brms package, version 2.14.4 (Bürkner, 2017), due to its flexibility in multilevel model specifications compared to other packages. All models fit with brm() used four Markov chains with 5000 iterations per chain. We retained the function's default (i.e. weakly informative) priors, given little previous work that specified the same or similar models that would otherwise have informed our beliefs about prior probabilities. Below, we report indirect associations, as these were most germane to the hypotheses we were testing. See Tables S5-S8 in the supplement for complete results from these models.

\section{Results}

\section{Overall descriptives}

Means, standard deviations, and zero-order correlations for all Level 1 (momentary) and Level 2 (person-level) variables are indicated in Table 1.

\section{Frequency of strategy use}

At the observation level, participants reported having desires $34 \%$ of the time. During desire episodes, they employed selfregulation strategies in the five above mentioned categories with variable frequency in the following order of popularity: cognitive strategies (30.1\%), distraction (26.2\%), suppression (18.3\%), situational/externally oriented strategies (9.8\%), and other (4.2\%).

Hypothesis 1: Strategy type For all models described below (by outcome measure), the following Level 1 variables were focal predictors: whether participants applied the following resistance strategies (or not): situational/externally-oriented strategies, distraction, cognitive strategies, or suppression, operationalized as separate, dummy-coded variables (to test efficacy of individual strategies) and as an ordinal variable (to test relative efficacy). As mentioned above, use of idiosyncratic strategies and momentary hunger were included as Level 1 covariates, as well as participants' age, BMI, and chronic dieting status at Level $2 .{ }^{3}$ Intercept terms in these models indicate the values of the outcome measures when no strategies were applied during a desire episode. Results of interest from models with strategy types as separate

\footnotetext{
${ }^{3}$ Note that in some of the reported results below, a few of the associations were weaker in unadjusted models, without any covariates (i.e., the association between cognitive strategies and desire enactment and amount eaten, and the association between suppression and desire enactment), which may be due to potential collinearity issues. However, since we pre-registered model specifications with a priori covariates, we only report from adjusted models.
}

Table 1 Summary statistics for all a priori variables of interest and covariates

\begin{tabular}{lc}
\hline Variable & Summary statistic \\
\hline Level 1 (observation-level) outcome measures & \\
Desire strength (SD) & $4.10(1.15)$ \\
Resistance (SD) & $1.56(1.79)$ \\
Desire enactment (\%) & $589(47.8 \%)$ \\
Amount eaten (SD) & $2.08(1.28)$ \\
Level 1 (observation-level) predictors and covariates & \\
Situational strategy (\%) & $125(9.8 \%)$ \\
Distraction (\%) & $333(26.2 \%)$ \\
Cognitive strategy (\%) & $383(30.1 \%)$ \\
Suppression (\%) & $232(18.3 \%)$ \\
Other strategy (\%) & $53(4.2 \%)$ \\
Number of strategies & \\
No strategies (\%) & $597(46.9 \%)$ \\
Single strategy (\%) & $344(27.0 \%)$ \\
Multiple strategies (\%) & $331(26.0 \%)$ \\
Hunger & $2.94(1.95)$ \\
Level 2 (person-level) covariates & $19.90(1.88)$ \\
Age (SD) & $23.40(3.02)$ \\
BMI in kg/m ${ }^{2}$ (SD) & $75(70.8)$ \\
Chronic dieting status (\%) & \\
\hline
\end{tabular}

For all continuous variables above, summary statistics are means and standard deviations, whereas for dichotomous variables the summary statistics are counts and percentages. NB: counts and percentages for level 1 variables are computed across all observations (i.e. not grouped by participant)

predictors are depicted in Fig. 1, while results from models with strategy type coded as an ordinal variable are depicted in Fig. 2. Complete results from these multilevel models are reported in the supplement (Tables S1 and S2, respectively).

\section{Efficacy of individual strategies}

Desire Strength Engaging in distraction was associated with significantly weaker desires, $b=-0.20,95 \%$ CI $[-0.35$, $-0.04], t=-2.46, p=0.014$. Applying situational/externally-oriented strategies was also associated with weaker desires, $b=-0.20,95 \%$ CI [:-0.41, 0.01], but this was not statistically significant, $t=-1.86, p=0.063$. No other strategies significantly predicted desire strength (all $p$ 's $\geq 0.14$; see Fig. 1 and Table S1 for complete model results).

Resistance All regulatory strategies were associated with higher levels of reported resistance, such that applying situational/externally-oriented strategies $(b=1.25,95 \% \mathrm{CI}$ [1.03, 1.47]), distraction $(b=1.27,95 \%$ CI [1.10, 1.43]), cognitive strategies ( $b=1.26,95 \% \mathrm{CI}[1.11,1.40])$, suppression $(b=1.27,95 \% \mathrm{CI}[1.09,1.46])$, and other/idiosyncratic 


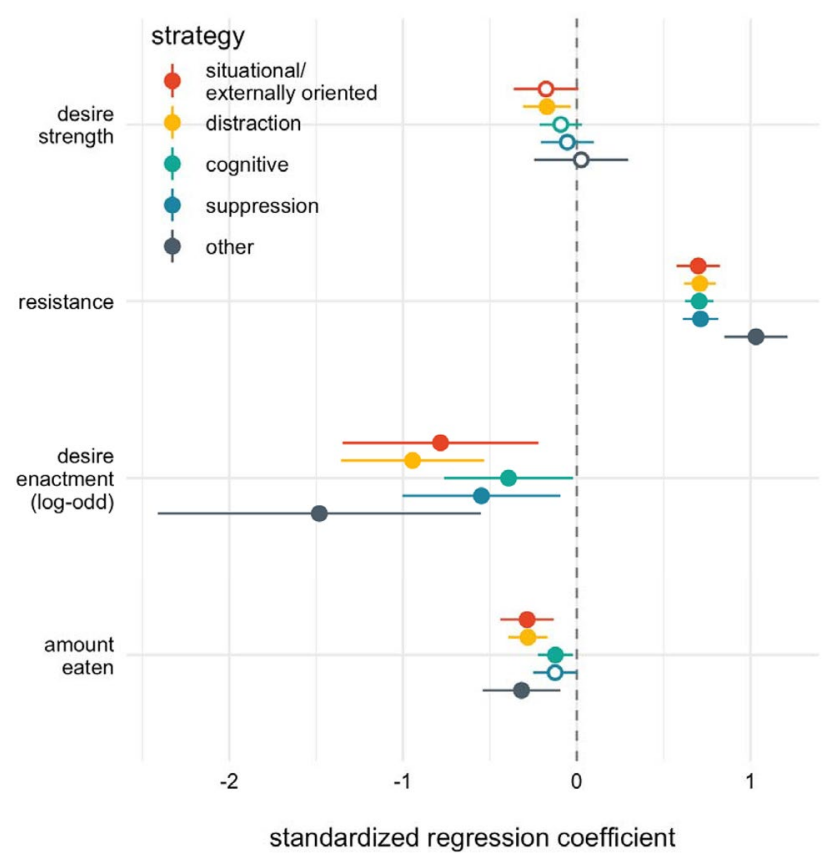

Fig. 1 Standardized regression coefficients for multiple regulatory strategies associated with the four outcomes of interest. NB: filled-in circles represent significant associations $(p<.05)$, whereas empty circles represent non-significant associations $(p>.05)$

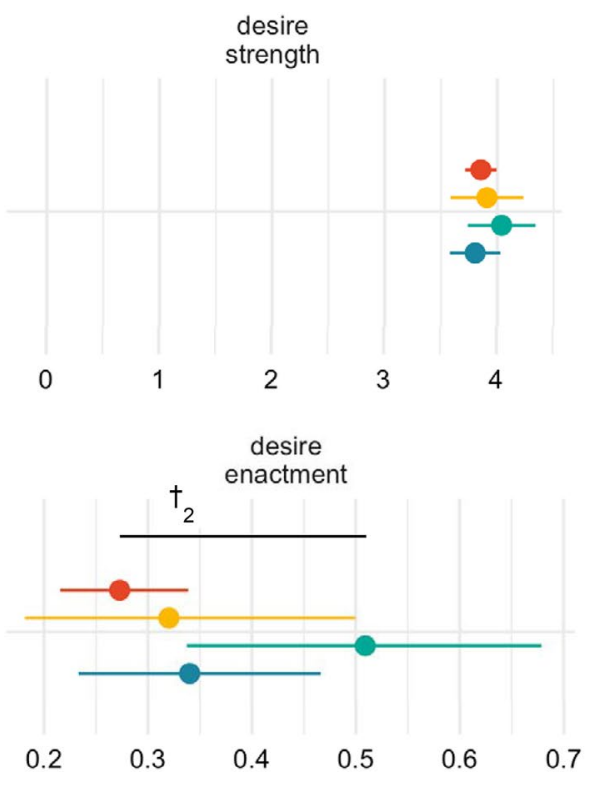

Fig. 2 Predicted values in models that tested for linear associations between use of particular strategy types and the four outcomes of interest. Black lines indicate that differences between strategies were tested, and subscript numbers refer to the respective contrasts run for strategies $(b=1.84,95 \%$ CI $[1.52,2.17])$ were associated with higher resistance, all $p$ 's $<0.001$.

Desire enactment As with resistance, all strategies were significantly associated with a lower likelihood of enacting reported desires to eat. This was true of situational/ externally-oriented strategies $\left(b_{\log }=-0.78, O R=0.46\right.$, 95\% CI [0.26, 0.80], $t=-2.73, p=0.006)$, distraction $\left(b_{\log }=-0.95, O R=0.39,95 \%\right.$ CI $[0.26,0.59], t=-4.50$, $p<0.001)$, cognitive strategies $\left(b_{\mathrm{log}}=-0.39, O R=0.68\right.$, $95 \%$ CI $[0.48,0.98], t=-2.07, p=0.038)$, suppression $\left(b_{\mathrm{log}}=-0.55\right.$, OR $=0.58,95 \%$ CI $[0.37,0.91]$, $t=-2.37, p=0.018)$, and other/idiosyncratic strategies $\left(b_{\log }=-1.48, O R=0.23,95 \%\right.$ CI $[0.09,0.58], t=-3.12$, $p=0.002)$.

Amount eaten Most strategies exhibited significant negative associations with the amount of food eaten during a given desire episode: situational/externallyoriented strategies $(b=-0.37,95 \%$ CI $[-0.56,-0.17]$, $t=-3.64, \quad p<0.001)$, distraction $(b=-0.36,95 \% \mathrm{CI}$ $[-0.50,-0.22]), t=-4.87, p<0.001$ cognitive strategies $(b=-0.16,95 \%$ CI $[-0.29,-0.03], t=-2.39$, $p=0.017)$, and other/idiosyncratic strategies $(b=-0.41$, 95\% CI $[-0.69,-0.12], t=-2.80, p=0.005)$. For sup-

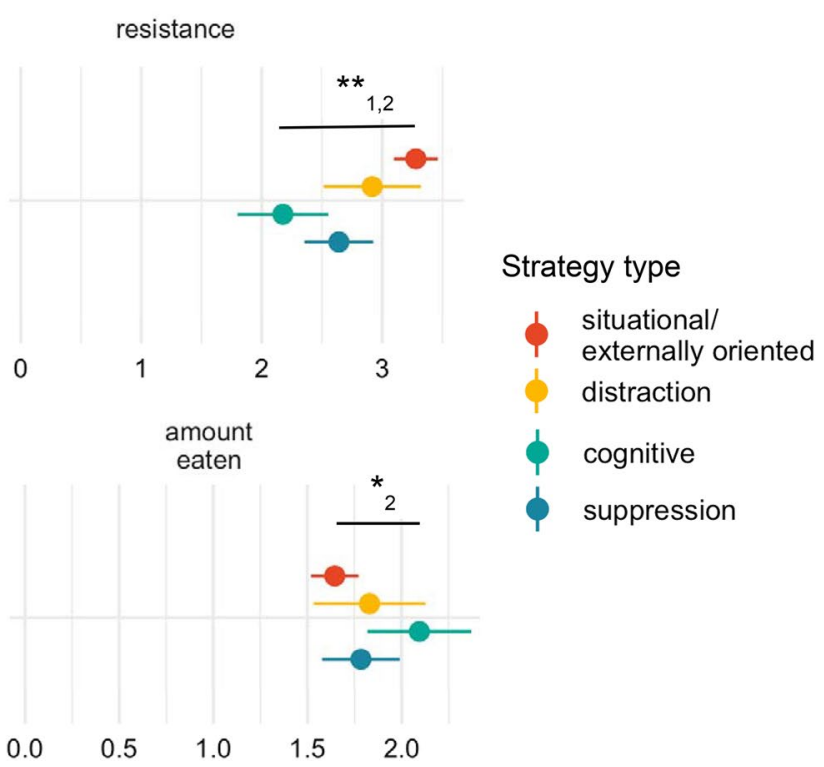

the strategy type variable $(1=$ linear contrast; $2=$ comparing situational/externally oriented strategies versus all others), and significance levels are as follows: $\dagger p=.075, * p<.05, * * p<.01$ 
pression, however, the negative relationship with amount eaten was not statistically significant, $b=-0.16,95 \% \mathrm{CI}$ $[-0.32,0.00], t=-1.94, p=0.053$.

\section{Relative efficacy of strategies as per the process model}

In additional model specifications with strategy use coded as an ordinal variable with a linear contrast, there was no linear trend observed for desire strength, $b=-0.01$, 95\% CI $[-0.26,-0.25], t=-0.04, p=0.968,{ }^{4}$ desire enactment, $b_{\log }=0.38, O R=1.46,95 \%$ CI $[0.80,2.68]$, $z=1.24, p=0.289$, or amount eaten, $b=0.15,95 \% \mathrm{CI}$ $[-0.08,0.39], t=1.26, p=0.289$. However, there was a significant linear trend for resistance, $b=-0.59,95 \% \mathrm{CI}$ $[-0.91,-0.28], t=-3.69, p=0.004$, such that participants tended to report more resistance with earlier strategies (see Fig. 2). Next, after applying the custom contrast comparing situational/externally-oriented strategies to all other strategies, there was no significant difference for desire strength, $b=0.06,95 \%$ CI $[-0.18,0.30], t=0.52, p=0.605$, but there was a significant difference for resistance, $b=0.70$, 95\% CI [1.00, 0.40], $t=4.63, p=0.004$, and amount eaten, $b=-0.26,95 \%$ CI $[-0.48,-0.04], t=-2.29, p=0.044$, such that there was greater resistance and less food eaten when participants applied situational/externally-oriented strategies versus other strategies (see Fig. 2). For desire enactment, the pattern was consistent with amount eaten, with lower likelihood of enactment when participants used situational/externally-oriented strategies versus others, but this was not statistically significant, $b_{\mathrm{log}}=0.56, O R=1.75$, $95 \%$ CI $[0.99,3.12], z=1.91, p=0.075$. Note that for the enactment model, BMI was removed as a covariate in order for the model to converge.

Hypothesis 2: Polyregulation Results of interest can be found in the coefficient plot in Fig. 3, and all results from these models are reported in Table S3. Applying additional strategies was associated with successful self-regulation of eating behaviors during the EMA period, as indicated by the following associations between the number of strategies participants reported using during a given desire episode and desire strength, $b=-0.14,95 \%$ CI $[-0.20,-0.07]$, $t=-4.00, p<0.001$, resistance, $b=1.25,95 \%$ CI $[1.17$, 1.32], $t=32.99, p<0.001$, desire enactment, $b_{\text {log }}=-0.64$, $O R=0.53,95 \%$ CI $[0.43,0.64], z=-6.65, p<0.001$, and

\footnotetext{
${ }^{4}$ Because these model specifications were not pre-registered, we report adjusted p-values to control for multiple tests and minimize type I error (false discovery rate) via the Benjamini and Hochberg (1995) procedure.
}

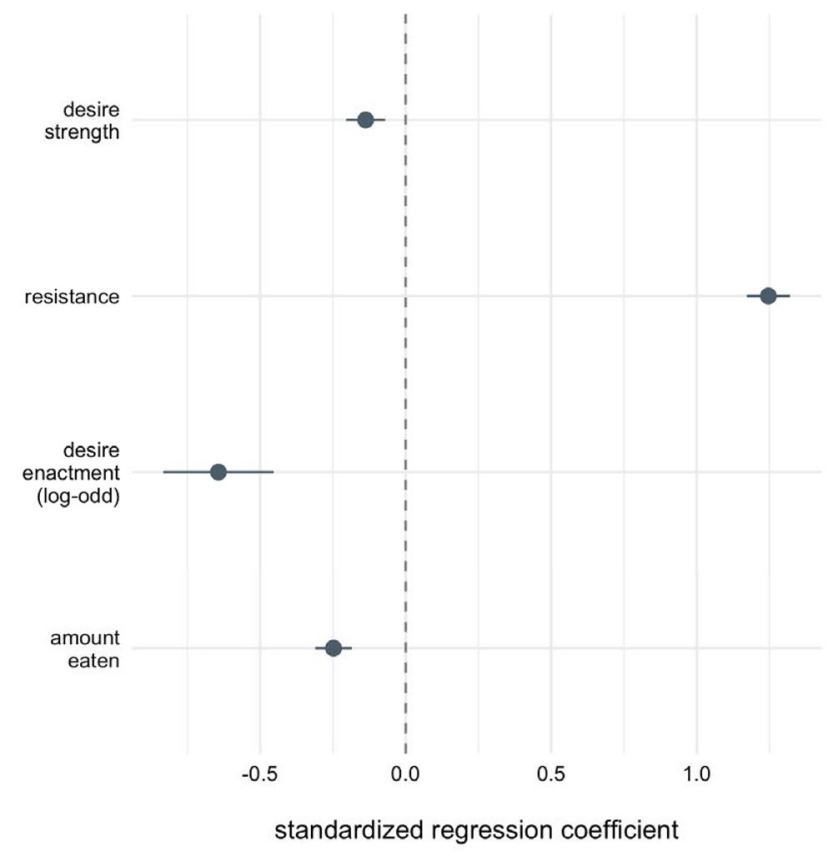

Fig. 3 Standardized regression coefficients from models that tested for associations between number of strategies applied (count variable) and the four outcome measures

amount eaten, $b=-0.25,95 \%$ CI $[-0.31,-0.19], t=-7.73$, $p<0.001$.

Hypothesis 3: Goal pursuit Across four multilevel models with participants' momentary experiences of goal conflict (or goal importance) as the predictor variables, number of strategies as the mediator, and desire enactment (or amount eaten) as the outcome measure, there were robust indirect associations, such that goal conflict and importance were associated with less frequent desire enactment and less food consumption, via additional regulatory strategies employed during desire episodes-all while controlling for participants' momentary hunger, dieting status, age, and body mass index (see Tables S5-S8 in supplement for all results). Specifically, higher goal conflict was associated with less frequent desire enactment (mean $b_{\log }=-0.20,95 \%$ Credible Interval $[-0.27,-0.14]$ ), as well as less food consumed (mean $b=-0.07,95 \%$ Credible Interval $[-0.10,-0.05]$ ), via number of employed strategies. This was also true of goal importance, with greater goal importance associated with less frequent desire enactment (mean $b_{\log }=-0.19$, 95\% Credible Interval $[-0.26,-0.13])$, as well as less food consumed (mean $b=-0.07,95 \%$ Credible Interval $[-0.09$, $-0.05]$ ), via number of employed strategies. 


\section{Discussion}

In the present study, we assessed the efficacy of multiple self-regulatory strategies on eating behaviors among college-aged women for one week. Overall, we found mixed evidence in support of our a priori hypotheses about associations between participants' use of specific self-regulatory strategies and their daily eating patterns. First, in preregistered model specifications that assessed efficacy of individual strategies while controlling for momentary hunger levels, chronic dieting status, and BMI, we observed that multiple strategies representing different stages of the process model (e.g. situational strategies, distraction, cognitive strategies) were individually effective, as operationalized by weaker desires, greater resistance, fewer desires enacted, and/or less food consumed. However, the relationships between strategy use and desire strength tended to be weaker and less consistent across strategies. This could be due to a number of reasons, but one possibility is that the experience of desire may temporally precede recognition of one's need to regulate (especially if the desire conflicts with other goals) and subsequent use of regulatory strategies. All four strategy categories (i.e. situational/externally oriented strategies, distraction, cognitive strategies, and suppression) were associated with greater resistance and less frequent desire enactment, and all strategies except suppression were associated with less food consumed.

This pattern of results is consistent with prior work that assessed self-regulatory strategies in an adolescent sample (De Vet et al., 2014) in that no strategy in the present sample clearly "won out" across the board. It is also worth noting that suppression, while showing relatively weaker effect sizes than other regulatory strategies/categories, was favorably associated with eating outcomes (e.g. weaker desires, less frequent enactment, etc.). This is somewhat contrary to hypotheses about strategies that involve response modulation being counterproductive, but is consistent with largescale cross-sectional studies (e.g. De Vet et al., 2014) as well as those that demonstrate the immediate effectiveness of response modulation (Johnston et al., 1999; Milyavskaya et al., 2020). However, it is noteworthy that several studies have indicated that, while individuals can suppress shortterm desires, such suppression leads to a re-bound effect on subsequent enactment opportunities (cf. Johnston et al., 1999). Thus, while not entirely in line with predictions of the process model, our findings are consistent with prior suggestions that suppression can be effective, at least for overriding desires in the short term.

Next, we explored the relative efficacy of the strategies based on predictions from the process model of self-control. We found that when participants resisted desires to eat, they generally exerted more resistance when employing earlier strategies (e.g. situational/externally oriented strategies and distraction) versus later strategies, as indicated by the significant linear contrast. When contrasting situational/externally oriented strategies versus all other strategies, we found that employing situational strategies tended to be associated with stronger resistance, eating less, and a lower likelihood of desire enactment, though this relationship was not statistically significant. Taken together, these results provide initial support for the hypothesis that participants would experience greater self-control success when employing more proactive strategies (e.g. situation selection) versus more reactive strategies (e.g. suppression). However, these model specifications directly comparing strategies were not preregistered and so these findings should be replicated. Moreover, given that we observed multiple strategies to be effective (in the preregistered model specification; see above), future research may benefit from employing a broader, "toolbox" approach to self-regulation training that highlights the utility and fit of each strategy (Fujita et al., 2020; Werner \& Ford, 2021), versus focusing exclusively on strategies hypothesized to be particularly effective, but not always practical (e.g. proactive situational strategies from the process model cannot always be implemented once people find themselves in specific situations). Lastly, we should note that these models used an ordinal variable that marked the earliest strategy (as per the process model) that participants used, so it did not explicitly account for use of additional strategies, as did our preregistered model with separate dummy-coded variables.

With regard to our second hypothesis informed by recent theorizing about polyregulation (Ford et al., 2019; Werner \& Ford, 2021), we found that when participants applied additional regulatory strategies during a desire episode, they experienced greater self-regulatory success across multiple metrics. Specifically, an increase in the number of applied strategies was associated with weaker desire strength, greater resistance, lower likelihood of desire enactment, and less food consumed. These results add some nuance to the findings reported thus far, as they suggest that it is not exclusively about which particular strategies are applied during a desire episode, effective though they may be, but the fact that they can be combined and used in tandem in order to promote successful goal pursuit. This suggests that polyregulation can also be an effective "meta-strategy" in the appetitive domain in the context of everyday food desires. Our results are also consistent with another recent EMA study in which using multiple self-regulation strategies was associated with reduced desire enactment across multiple domains (Milyavskaya et al., 2020). Taken together, these results suggest not only that diverse self-regulation strategies are effective, but that their effectiveness may increase in an additive manner as more strategies are applied to a single desire episode. 
Lastly, to test our hypothesis about relationships between participants' momentary experiences of goal-related processes and self-regulatory success, we ran a set of multilevel mediation models to test whether goal conflict and goal importance were associated with successful goal pursuit, via the number of strategies applied during a given desire episode. We observed reliable indirect associations between the hypothesized goal variables and the primary outcome variables (desire enactment and amount eaten). For example, for those desire episodes in which participants reported their desires as conflicting with other goals they had, the less likely they were to enact those desires, and this relationship was partially mediated by polyregulation (i.e. the number of strategies applied) during those episodes.

This suggests that successful goal pursuit in the eating domain, as indicated by less frequent enactment and less food consumed, may be marked by goal-related processes (i.e. goal conflict and goal importance), which can serve as a signal in that moment to engage in polyregulation. This pattern is consistent with previous theorizing about the importance of these processes (Kotabe \& Hofmann, 2015), and it also suggests that when a person can bring to mind selfregulatory goals and their importance, in the moment, it may motivate them to apply additional strategies that increase the likelihood of success. Also, the fact that participants applied these strategies spontaneously, without specific instructions to regulate, hints that they may have been more autonomously motivated to pursue goals that conflicted with their desires. But this possibility cannot be confirmed with the present data and would have to be explicitly tested in future studies.

Taken together, the present study adds to growing body empirical work on the ecological validity of self-regulatory strategies (Blanke et al., 2020; Burr \& Samanez-Larkin, 2020; Southward \& Cheavens, 2020; Williamson \& Wilkowski, 2020) and demonstrates the efficacy of self-regulatory strategies when applied to daily food desires that at times conflict with people's goal pursuit. Indeed, the current findings shed light on the domain specificity of these strategies as they are applied to desires to eat in particular, versus desires that people experience across domains (Milyavskaya et al., 2020). In our mediation analyses, we further included specific variables targeting goal processes (conflict and importance) to investigate desire episodes in which people are motivated to regulate goal-incongruent desires (versus resisting desires more generally, as in Milyavskaya et al., 2020). Here, we found that polyregulation was particularly elevated at moments of high goal conflict (i.e. when selfcontrol is needed most; Inzlicht et al., 2020; Werner \& Ford, 2021 ), and that this in turn was associated with greater regulation. As such, the inclusion of measures of goal processes (conflict and importance) are critically important for ongoing work investigating polyregulation, perhaps because such measures capture variance (i.e. conflict between competing alternatives) that is foundational to self-control itself.

Despite the merits of the present work and the fact that some of the findings were consistent with our a priori hypotheses, there are several limitations worth mentioning. First, although all preregistered models tested directional hypotheses, the EMA data were correlational; that is, all variables were assessed concurrently for each EMA signal. Given that the experience of a food desire unfolds over time, some variables presumably precede others (e.g. strength of a desire, resistance, and strategies applied to a desire will precede enactment of the desire and eating). However, this does not mitigate the issues that no strong causal claims can be made about the variables of interest. For example, while it could be that polyregulation (i.e. applying additional strategies) decreases eating over time, it could also be the case that those individuals who do not enact their food desires as frequently have more readily available cognitive and attentional resources they can utilize in order to engage in polyregulation. Additionally, recognition and/or use of some strategies might gradually become more automatic (e.g. developing a habit of avoiding particular situations or contexts that thwart one's goal pursuit), so a longitudinal design could elucidate changes in strategy use and habits over time.

And although there is theoretical justification for the ordering of variables in the mediation models reported here (i.e. goal-related processes as $\mathrm{X}$, polyregulation as $\mathrm{M}$, and eating behaviors as $\mathrm{Y}$ ), we caution the reader against forming any definitive conclusions about the nature and strength of the observed indirect associations. We mention this because of known issues that can arise from mediation models fit with cross-sectional data, including biased estimates of indirect effects (Maxwell \& Cole, 2007) and general threats to validity and inference (Kenny, 2019). Future studies that employ longitudinal designs or specify multilevel models that explicitly test for lagged associations would be better suited to establish reliable estimates of indirect paths, and thus stronger inferences about causality between the variables of interest. We also recommend that future research employs more finer-grained assessment of key constructs of interest, especially resistance. Although we observed robust associations between strategy type and resistance, the measure of resistance was always the same regardless of strategy and non-specific, so it is not clear why some strategies required more resistance than others, and what exactly resistance for each strategy entailed.

Another limitation is that we only recruited college-aged women to participate in this study, so the present findings may not generalize beyond this population. This was due to the eligibility criteria of the original studies for which the EMA data were collected, as well as to avoid the confound of sex-related effects on eating behaviors among college-aged 
men and women (Holm-Denoma et al., 2008). Lastly, while there was some racial and ethnic diversity in our sample, it consisted mostly of White participants enrolled at an elite private college, so any findings about the efficacy of these regulatory strategies would need to be replicated in more heterogeneous samples along racial, ethnic, and socio-economic dimensions.

To conclude, the present study builds on prior work to further establish the ecological validity of models of selfcontrol (e.g. Hennecke et al., 2019; Hofmann et al., 2012; Williamson \& Wilkowski, 2020), that focus on how people exert control, uninstructed, in naturalistic contexts versus laboratory settings. Here, we tested predictions about eating behavior derived from the process model of self-control in a sample of college-aged women. Overall, our hypotheses were partially supported. First, it would seem that using any regulatory strategy can be indeed effective, as indicated by associations we observed with multiple outcome measures of interest (e.g. higher resistance, less frequent desire enactment), and that particular strategies may be relatively more efficacious in some cases (e.g. proactive/situational strategies, whenever possible to implement, may be particularly effective). Additionally, polyregulation (i.e. applying more than one strategy during a desire episode) was not only associated with self-regulatory success, but it was a metastrategy of choice during desire episodes in which participants reported goals that were at odds with the food desires they experienced. Future work can extend various aspects of these findings. For example, one potentially fruitful direction could focus on regulatory training over time that encourages polyregulation of eating behaviors in tandem with goal setting and goal adherence. If promising findings are obtained in the eating domain, then researchers can examine these questions in other domains that call for self-regulation, from alcohol and illicit drugs to patterns of compulsive social media use.

Supplementary Information The online version contains supplementary material available at https://doi.org/10.1007/s11031-021-09903-4.

Acknowledgements We would like to thank Niklas Johannes for his thoughtful and constructive feedback on an earlier version of this manuscript.

Open Access This article is licensed under a Creative Commons Attribution 4.0 International License, which permits use, sharing, adaptation, distribution and reproduction in any medium or format, as long as you give appropriate credit to the original author(s) and the source, provide a link to the Creative Commons licence, and indicate if changes were made. The images or other third party material in this article are included in the article's Creative Commons licence, unless indicated otherwise in a credit line to the material. If material is not included in the article's Creative Commons licence and your intended use is not permitted by statutory regulation or exceeds the permitted use, you will need to obtain permission directly from the copyright holder. To view a copy of this licence, visit http://creativecommons.org/licenses/by/4.0/.

\section{References}

Arend, M. G., \& Schäfer, T. (2019). Statistical power in two-level models: A tutorial based on Monte Carlo simulation. Psychological Methods, 24(1), 1-19.

Bates, D., Mächler, M., Bolker, B. M., \& Walker, S. C. (2014). Fitting linear mixed-effects models using lme4. Journal of Statistical Software. https://doi.org/10.18637/jss.v067.i01

Baumeister, R. F., Bratslavsky, E., Muraven, M., \& Tice, D. M. (1998). Ego depletion: Is the active self a limited resource? Journal of Personality and Social Psychology, 74(5), 1252-1265.

Blanke, E. S., Brose, A., Kalokerinos, E. K., Erbas, Y., Riediger, M., \& Kuppens, P. (2020). Mix it to fix it: Emotion regulation variability in daily life. Emotion, 20(3), 473-485.

Bürkner, P.-C. (2017). brms: An R package for Bayesian multilevel models using Stan. Journal of Statistical Software, Articles, 80(1), $1-28$.

Burr, D. A., \& Samanez-Larkin, G. R. (2020). Advances in emotionregulation choice from experience sampling. Trends in Cognitive Sciences. https://doi.org/10.1016/j.tics.2020.02.008

Cosme, D., \& Lopez, R. B. (2020). Neural indicators of food cue reactivity, regulation, and valuation and their associations with body composition and daily eating behavior. Social Cognitive and Affective Neuroscience. https://doi.org/10.1093/scan/nsaa1 55

Dang, J., Barker, P., Baumert, A., Bentvelzen, M., Berkman, E., Buchholz, N., \& Zinkernagel, A. (2020). A Multilab Replication of the Ego Depletion Effect. Social Psychological and Personality Science. https://doi.org/10.1177/1948550619887702

De Vet, E., De Ridder, D., Stok, M., Brunso, K., Baban, A., \& Gaspar, T. (2014). Assessing self-regulation strategies: Development and validation of the tempest self-regulation questionnaire for eating (TESQ-E) in adolescents. The International Journal of Behavioral Nutrition and Physical Activity, 11, 106.

Duckworth, A. L., Gendler, T. S., \& Gross, J. J. (2016). Situational strategies for self-control. Perspectives on Psychological Science: A Journal of the Association for Psychological Science, 11(1), $35-55$.

Duckworth, A. L., Milkman, K. L., \& Laibson, D. (2018). Beyond willpower: Strategies for reducing failures of self-control. Psychological Science in the Public Interest: A Journal of the American Psychological Society, 19(3), 102-129.

Ford, B. Q., Gross, J. J., \& Gruber, J. (2019). Broadening our field of view: The role of emotion polyregulation. Emotion Review: Journal of the International Society for Research on Emotion, 11(3), 197-208.

Friedrich, M. J. (2017). Global obesity epidemic worsening. JAMA: The Journal of the American Medical Association, 318(7), 603.

Friese, M., Loschelder, D. D., Gieseler, K., Frankenbach, J., \& Inzlicht, M. (2018). Is ego depletion real? An analysis of arguments. Personality and Social Psychology Review: An Official Journal of the Society for Personality and Social Psychology, Inc. https:// doi.org/10.1177/1088868318762183

Fujita, K. (2011). On conceptualizing self-control as more than the effortful inhibition of impulses. Personality and Social Psychology Review An Official Journal of the Society for Personality and Social Psychology Inc, 15(4), 352-366.

Fujita, K., Orvell, A., \& Kross, E. (2020). Smarter, not harder: A toolbox approach to enhancing self-control. Policy Insights from the Behavioral and Brain Sciences, 7(2), 149-156.

Gillebaart, M., \& de Ridder, D. T. D. (2015). Effortless self-control: A novel perspective on response conflict strategies in trait selfcontrol: Effortless self-control. Social and Personality Psychology Compass, 9(2), 88-99. 
Giner-Sorolla, R., Aberson, C. L., Bostyn, D. H., Carpenter, T., Conrique, B. G., Lewis, N. A., \& Soderberg, C. (2019). Power to detect what? Considerations for planning and evaluating sample size. Unpublished Manuscript. Retrieved from https://osf.io/ jnmya/download

Gollwitzer, P. M. (1999). Implementation intentions: Strong effects of simple plans. The American Psychologist, 54(7), 493.

Grommisch, G., Koval, P., Hinton, J. D. X., Gleeson, J., Hollenstein, T., Kuppens, P., \& Lischetzke, T. (2019). Modeling individual differences in emotion regulation repertoire in daily life with multilevel latent profile analysis. Emotion. https://doi.org/10.1037/ emo0000669

Hagger, M. S., Chatzisarantis, N. L. D., Alberts, H., Anggono, C. O., Batailler, C., Birt, A. R., \& Zwienenberg, M. (2016). A multilab preregistered replication of the ego-depletion effect. Perspectives on Psychological Science: A Journal of the Association for Psychological Science, 11(4), 546-573.

Heatherton, T. F., Herman, C. P., Polivy, J., King, G. A., \& McGree, S. T. (1988). The (mis)measurement of restraint: An analysis of conceptual and psychometric issues. Journal of Abnormal Psychology, 97(1), 19-28.

Heatherton, T. F., Polivy, J., \& Herman, C. P. (1991). Restraint, weight loss, and variability of body weight. Journal of Abnormal Psychology, 100(1), 78-83.

Hennecke, M., \& Bürgler, S. (2020). Many roads lead to Rome: Selfregulatory strategies and their effects on self-control. Social and Personality Psychology Compass, 14(6), 396.

Hennecke, M., Czikmantori, T., \& Brandstätter, V. (2019). Doing despite disliking: Self-regulatory strategies in everyday aversive activities. European Journal of Personality, 33(1), 104-128.

Herman, C. P., \& Polivy, J. (1980). Restrained eating. In A. J. Stunkard (Ed.), Obesity (pp. 208-225). Saunders.

Hofmann, W. (2017). Working memory capacity and self-control. In Denise de Ridder, Marieke Adriaanse, \& Kentaro Fujita (Eds.), Routledge International Handbook of Self-Control in Health and Well-Being. Routledge.

Hofmann, W., Baumeister, R. F., Förster, G., \& Vohs, K. D. (2012). Everyday temptations: An experience sampling study of desire, conflict, and self-control. Journal of Personality and Social Psychology, 102(6), 1318-1335.

Hofmann, W., \& Kotabe, H. (2012). A general model of preventive and interventive self-control. Social and Personality Psychology Compass, 6(10), 707-722.

Holm-Denoma, J. M., Joiner, T. E., Vohs, K. D., \& Heatherton, T. F. (2008). The "freshman fifteen" (the "freshman five" actually): Predictors and possible explanations. Health Psychology: Official Journal of the Division of Health Psychology, American Psychological Association, 27(1S), S3-9.

Inzlicht, M., Werner, K. M., Briskin, J. L., \& Roberts, B. W. (2020). Integrating models of self-regulation. Annual Review of Psychology. https://doi.org/10.1146/annurev-psych-061020-105721

Jebb, S. A., Cole, T. J., Doman, D., Murgatroyd, P. R., \& Prentice, A. M. (2000). Evaluation of the novel Tanita body-fat analyser to measure body composition by comparison with a four-compartment model. The British Journal of Nutrition, 83(2), 115-122.
Johnston, L., Bulik, C. M., \& Anstiss, V. (1999). Suppressing thoughts about chocolate. The International Journal of Eating Disorders, 26(1), 21-27.

Kenny, D. A. (2019). Enhancing validity in psychological research. The American Psychologist, 74(9), 1018-1028.

Kotabe, H. P., \& Hofmann, W. (2015). On integrating the components of self-control. Perspectives on Psychological Science: A Journal of the Association for Psychological Science, 10(5), 618-638.

Lopez, R. B., Chen, P.-H.A., Huckins, J. F., Hofmann, W., Kelley, W. M., \& Heatherton, T. F. (2017). A balance of activity in brain control and reward systems predicts self-regulatory outcomes. Social Cognitive and Affective Neuroscience, 12(5), 832-838.

Lopez, R. B., Hofmann, W., Wagner, D. D., Kelley, W. M., \& Heatherton, T. F. (2014). Neural predictors of giving in to temptation in daily life. Psychological Science, 25(7), 1337-1344.

Lopez, R. B., Milyavskaya, M., Hofmann, W., \& Heatherton, T. F. (2016). Motivational and neural correlates of self-control of eating: A combined neuroimaging and experience sampling study in dieting female college students. Appetite, 103, 192-199.

Maxwell, S. E., \& Cole, D. A. (2007). Bias in cross-sectional analyses of longitudinal mediation. Psychological Methods, 12(1), 23-44.

Metcalfe, J., \& Mischel, W. (1999). A hot/cool-system analysis of delay of gratification: Dynamics of willpower. Psychological Review, 106(1), 3.

Milyavskaya, M., Saunders, B., \& Inzlicht, M. (2020). Self-control in daily life: Prevalence and effectiveness of diverse self-control strategies. Journal of Personality. https://doi.org/10.1111/jopy. 12604

Milyavskaya, M., \& Werner, K. M. (2018). Goal pursuit: Current state of affairs and directions for future research. Canadian Psychology/ psychologie Canadienne, 59(2), 163-175.

O’Leary, D., Suri, G., \& Gross, J. J. (2018). Reducing behavioural risk factors for cancer: An affect regulation perspective. Psychology \& Health, 33(1), 17-39.

Patti, M.-E., \& Kahn, B. B. (2004). Nutrient sensor links obesity with diabetes risk. Nature Medicine, 10(10), 1049-1050.

Rodriguez, M. L., Mischel, W., \& Shoda, Y. (1989). Cognitive person variables in the delay of gratification of older children at risk. Journal of Personality and Social Psychology, 57(2), 358-367.

Southward, M. W., \& Cheavens, J. S. (2020). More (of the right strategies) is better: Disaggregating the naturalistic between- and within-person structure and effects of emotion regulation strategies. Cognition \& Emotion, 34(8), 1729-1736.

Wagner, D. D., Altman, M., Boswell, R. G., Kelley, W. M., \& Heatherton, T. F. (2013). Self-regulatory depletion enhances neural responses to rewards and impairs top-down control. Psychological Science, 24(11), 2262-2271.

Werner, K. M., \& Ford, B. Q. (2021). Self-control: An integrative framework. Doi:https://doi.org/10.31234/osf.io/y6kxa

Williamson, L. Z., \& Wilkowski, B. M. (2020). Nipping temptation in the bud: examining strategic self-control in daily life. Personality \& Social Psychology Bulletin, 46(6), 961-975.

Publisher's Note Springer Nature remains neutral with regard to jurisdictional claims in published maps and institutional affiliations. 